\title{
Honing a harder-hitting hammerhead improves broadly neutralizing antibody breadth and potency
}

\author{
George K. Lewis
}

Division of Vaccine Research, Institute of Human Virology, University of Maryland School of Medicine, Baltimore, Maryland, USA.

\begin{abstract}
While current HIV-1 therapies have greatly improved the quality and duration of life for infected individuals, a vaccine to prevent transmission of the virus is lacking. Broadly neutralizing monoclonal antibodies (bnmAbs) with the capacity to neutralize multiple HIV-1 variants have been isolated from HIV-1-infected individuals, and there has been a great effort to investigate how these bnmAbs arise, due their potential for HIV-1 vaccination. In this issue of the $J C l$, Willis and colleagues apply a computational approach to design variants of the bnmAb PG9 in an attempt to enhance potency and neutralization breadth. One of these variants was able to target multiple PC9-resistant strains, as the result of stabilization of the long heavy chain complementarity determining region 3 (HCDR3). The results of this study provide important insight and a unique approach to optimizing HIV-1 bnmABs.
\end{abstract}

receptor-binding domain (gp120) and a membrane-anchored domain (gp41) that mediates membrane fusion (reviewed in ref. 13). Neutralization-fit bnmAbs have been identified that recognize gp120 epitopes, including the V1/V2 region plus glycans (defined by PG9; refs. 14, 15), the V3 region plus glycans (defined by PGT121; ref. 16), the outer domain (OD) plus glycans (defined by 2G12; ref. 17), and the CD4 binding site (CD4BS; defined by VRC01; ref. 18). Other neutralization-fit bnmAbs recognize gp41 epitopes in the membrane proximal region (MPER), and this group is defined by several classes of bnmAbs, including 2F5, 4E10, and 10E8 (reviewed in ref. 4), with 10E8 being far and away the most neutralization fit (19). Additionally, neutralization-fit bnmAbs can recognize hybrid epitopes comprising elements of both gp120 and gp41 - defined by bnmAbs PGT151 (20), 8 ANC195 (21), and $35 \mathrm{O} 22$ (22). Thus, there is no shortage of potential epitope targets for neutralization-fit bnmAbs; however, the issue remains as to how to generate such antibodies with a vaccine.

Unfortunately, neutralization-fit bnmAbs have only been observed in HIV-1infected people (3) and SIV-infected rhesus macaques (23); these bnmAbs are not detectable until approximately $21 / 2$ years (24) and two-thirds of a year (23) after infection, respectively. The convergence of several lineage studies indicates that neutralization-fit bnmAbs arise only in response to exposure to different viral variants over these time periods $(6,7)$. Thus, the emergence of bnmAbs is the result of a predator-prey interaction in which the bnmAbs become increasingly "fit" in response to the increased viral variation that emerges in response to antibody pressure. Many of the identified pathways to neutralization fitness differ among studies, and it is not yet clear whether these pathways can be recapitulated by vaccination. Currently, it appears that lengthy (and clinically cumbersome) immuniza-
Conflict of interest: The author owns shares in Profectus BioSciences, which is developing vaccines against HIV- 1 and is a sub-contractor on NIH Small Business Innovative Research grants awarded to Profectus BioSciences; otherwise, there are no consulting or financial arrangements with the company. This conflict is managed by the University of Maryland Baltimore pursuant to state and federal requirements.

Reference information: / Clin Invest. 2015;125(6):2271-2274. doi:10.1172/JCI82057. 


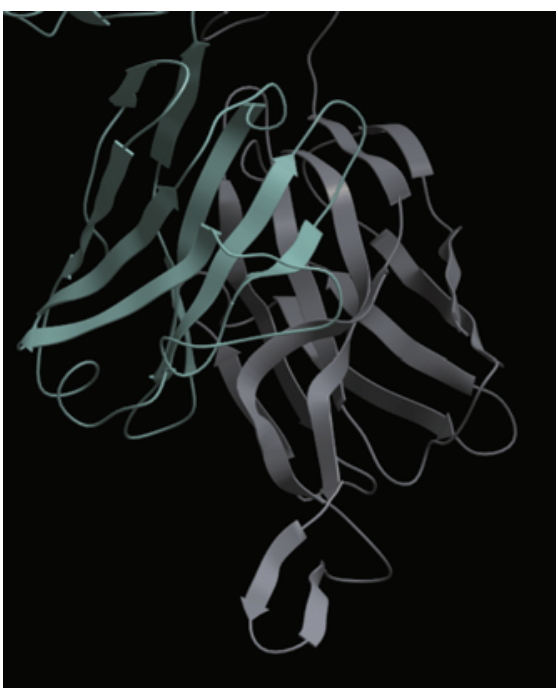

tion schedules will be required to elicit neutralization-fit bnmAbs by a vaccine. This problem is confounded further by the likely need for multiple variants of Env in trimer immunogens to drive neutralization fitness. Solving this problem is a tall order, but there is hope that some pathways to neutralization fitness are shorter than others. Such appears the case for the PG9 class of bnmAbs.

\section{PG9: navigating the road to neutralization fitness}

bnmAbs are typically characterized either by high levels of somatic hypermutation, long heavy chain complementarity determining region 3 (HCDR3) lengths, or both, regardless of epitope specificity (reviewed in refs. 4, 6, 7). HCDR3 lengths upwards of 25 residues are characteristic of glycan-shield and anti-MPER antibodies (reviewed in refs. 4, 6, 7), with high frequencies of somatically mutated residues also present in some of these glycan-shield and anti-MPER bnmAbs (reviewed in refs. $4,6,7)$. In contrast, CD4BS antibodies typically have moderate-length HCDR3s but are highly mutated, and the mutation of framework residues even contributes in unexpected ways to specificity (ref. 25; reviewed in refs. $4,6,7$ ). It is unclear how these disparate pathways to neutralization fitness can be mimicked by vaccination, but in the case of the PG9 series, it is possible that the road to neutralization fitness may be the shortest.

Unlike some bnmAbs, the PG9 series does not require high levels of somatic hypermutation to achieve neutralization
Figure 1. Depiction of the PC9 HCDR3 hammerhead. The Fv region of PC9 is shown with the heavy chain in gray and the light chain in cyan. Prominent extension of the hammerhead from the antigen-binding face of the Fv structure is readily apparent at the bottom of the figure. The figure was made with the ICM software suite (Molsoft LLC., La Jolla, California, USA) using PDB: 3u4e from ref. 13.

fitness $(1,14,15,26)$. Rather, these bnmAbs use long HCDR3s - which are often tyrosine-sulfated - to penetrate the glycan shield, thereby achieving neutralization fitness. Long HCDR3 lengths are determined by rearrangements of certain $\mathrm{D}$ and $\mathrm{J}$ segments during B cell development and not by insertional mutagenesis during somatic hypermutation (27). If an immunogen can be found that stimulates primary $B$ cells that are specific for the PG9 bnmAb series, it is likely that a protracted immunization schedule will not be necessary to achieve neutralization fitness. Willis and collaborators shed new light on how the PG9 series of bnmAbs reach neutralization fitness without extensive somatic hypermutation; the authors demonstrated that a point mutation in HCDR3 can markedly increase the neutralization potency and breadth of PG9 (8). While it may not be surprising that a single mutation can improve the neutralization fitness of PG9, the way in which Willis et al. identified this mutation and how it increases neutralization fitness provides important insight into improving bnmAb function.

\section{A harder-hitting hammerhead}

The long HCDR3s found in the PG9 series of bnmAbs results in an unusual hammerhead structure (Figure 1; refs. 14, 15, 26) that is stabilized by a network of hydrogen bonds that extends from the face of the antigen-binding fragment (Fab) and contacts both glycans and residues of the V1/ V2 region of gp120 (Figure 2). bnmAb binding at this region of gp120 involves glycan, electrostatic, and sequence-independent interactions, each of which appears to be critical for function $(14,15,26)$. Furthermore, PG9 makes contact with two protomers in the Env trimer, resulting in the unusual stoichiometry of $1 \mathrm{Fab}$ per trimer (28), in contrast to the usual 3:1 Fab-totrimer ratio for other neutralization-fit bnmAbs $(11,12,28,29)$. Another hallmark of PG9 specificity is that this bnmAb only neutralizes viruses that have particular glycan at N160 in the V1/V2 region (Figure 2; reviewed in ref. 4). Based on these properties, PG9 was a sound choice for rational improvement, since most of its interactions with antigens are due to HCDR3; however, this choice has its own difficult experimental problem.

The PG9 HCDR3 is 28-residues long; therefore, there are 532 possible singlepoint mutations, and up to $2 \times 10^{29}$ possible variants accounting for multiple-point mutations. The effort required to construct and screen this point mutation and resulting variants is out of reach without further winnowing of the possibilities. Willis and colleagues turned to the Rosetta Design software suite (reviewed in ref. 30) to solve this problem (8). The PG9 CDHR3 sequence was redesigned computationally to optimize its thermodynamic stability with a predicted increase in binding energy (based on the starting structure in ref. 13). Willis et al. used these computations, coupled with visual inspection of energetically favored residues that might alter function, to whittle down the possible designs down to three single-point mutations, $\mathrm{N} 100_{\mathrm{F}} \mathrm{Y}, \mathrm{N} 100_{\mathrm{F}} \mathrm{L}$, and $\mathrm{D} 100_{\mathrm{L}} \mathrm{N}$, as well as two other variants with either two or four mutations (8). This is an example of the power of Rosetta Design to rationally reduce the number of candidate structures for in-depth analysis of structure and function. Of these variants, PG9_N100 F exhibited the most consistent increases in binding and neutralization fitness for all viruses tested. Strikingly, this variant also neutralized viruses that lacked a glycosylation site at position 160. Neutralization fitness was also increased for PG9_N100 $\mathrm{F}$, but the improvements for this PG9 variant were less than those for PG9_N100 Y. Thus, a single-point mutation at $\mathrm{N} 100_{\mathrm{F}} \mathrm{Y}$ dramatically increased the neutralization fitness of PG9, suggesting that such variants could arise without the extensive mutational gymnastics required for neu- 


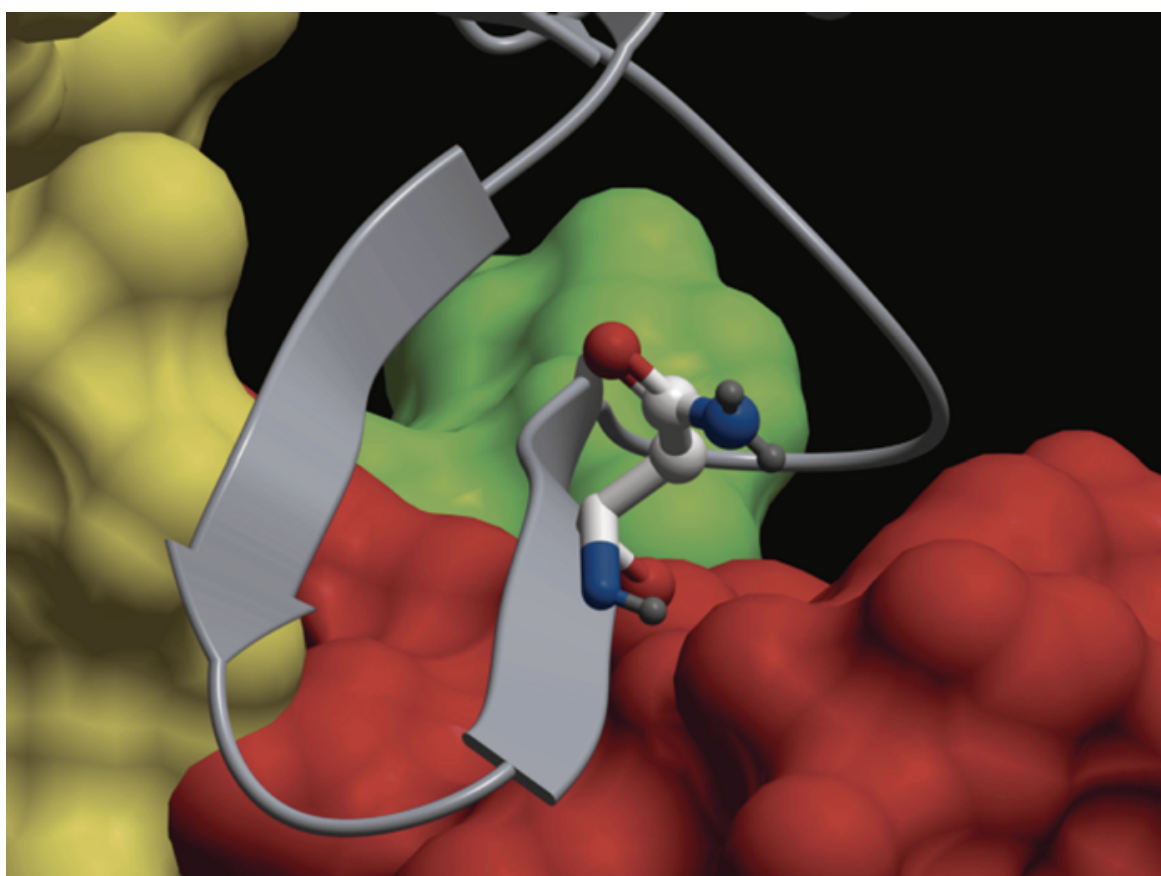

tralization fitness for the other classes of bnmAbs. This result strongly suggests that a vaccine that targets the epitopes recognized by the PG9 class of bnmAbs could obviate the need for a lengthy immunization schedule. The early emergence of PG9-like bnmAbs during HIV-1 infection also provides hope on this front (31).

\section{Conclusions and future directions}

In addition to vaccine implications, the study by Willis and colleagues also provides a template for the rational improvement of other bnmAbs for prevention and treatment of a variety of diseases. Interestingly, the PG9_N100_Y mutation does not alter a contact residue (Figure 2). Instead, the replacement of the carboxamide side chain of asparganine with the phenol of tyrosine preserved the overall structure of the paratope. Willis and colleagues directly confirmed that the $\mathrm{N} 100_{\mathrm{F}} \mathrm{Y}$ mutation in the HCDR3 of PG9 increased thermodynamic stability of the hammerhead, which most likely forces the binding mechanism to be more of a lock and key than an induced fit. Thus, it is possible that neutralization fitness can be increased for other classes of bnmAb by redesigning paratope residues to increase thermodynamic stability. The identification and characterization of PG9_N100 F mutation also speaks to a final problem for generat- ing neutralization-fit bnmAbs with a vaccine. It is known that long HCDR3 usage is often associated with autoimmune diseases in which auto-reactive B cells escape tolerance checkpoints during B cell development. A corollary of long HCDR3 usage is that naive $\mathrm{B}$ cells specific for bnmAb epitopes might be deleted during normal B cell development if they are auto-reactive. This appears to be the case for certain anti-MPER bnmAbs (reviewed in ref. 32). Although PG9 has been reported to be poly-reactive, it is not auto-reactive (33), suggesting that B cell precursors specific for this epitope class will not be deleted at tolerance checkpoints. Fortunately, the PG9_N100 Y mutation did not cause PG9 to become auto-reactive, suggesting that the PG9 class of bnmAbs has a clear path to neutralization potency without facing clonal deletion or the need for high levels of somatic hypermutation.

In summary, Willis and colleagues report a novel approach for querying a bnmAb class to determine whether neutralization fitness can be improved, and they predict whether this improvement will require extensive somatic hypermutation. Thus, through a simple, computationally predicted point mutation, Willis et al. have honed a harder-hitting hammerhead for PG9 that increases neutralization fitness. Such an approach for improving neutralization fitness will be of considerable
Figure 2. Hammerhead binding to the PG9 epitope. The hammerhead is shown as a gray ribbon with $\mathrm{N} 100_{\mathrm{F}}$ shown as ball and sticks. The glycan at N160 is shown as a yellow surface, the glycan at N156 is shown as a green surface, and V1/V2 is shown as a red surface. HCDR3 contacts all three structures where the most prominent are with the glycan at N160 (further details in ref. 13). The carboxamide side chain of N100F does not contact any of the epitope residues. The figure was generated as in Figure 1 using PDB:3u4e (13) and PDB:4tvp (10).

importance for future HIV-1 vaccine development, as well as for the development of prophylactic and therapeutic bnmAbs to prevent or treat a variety of infections.

\section{Acknowledgments}

The author thanks his Institute of Human Virology colleagues Anthony L. DeVico, Marzenza Pazgier, Mohammad Sajadi, Krishanu Ray, Roberta Kamin-Lewis, Alan Schmaljohn, and Robert C. Gallo for their contributions to our ongoing work to understand antibody-mediated protection against HIV. This work was supported by grants OPP1017606 and OPP1033109 from the Bill and Melinda Gates Foundation and by R01AI087181 from the National Institute of Allergy and Infectious Diseases (NIAID), NIH.

Address correspondence to: George K. Lewis, Division of Vaccine Research, Institute of Human Virology, University of Maryland School of Medicine, 725 West Lombard Street, Baltimore, Maryland 21201, USA. Phone: 443.540.0393; E-mail: glewis@ihv.umaryland.edu.

1. Walker LM, et al. Broad and potent neutralizing antibodies from an African donor reveal a new HIV-1 vaccine target. Science. 2009;326(5950):285-289.

2. Simek MD, et al. Human immunodeficiency virus type 1 elite neutralizers: individuals with broad and potent neutralizing activity identified 
by using a high-throughput neutralization assay together with an analytical selection algorithm. J Virol. 2009;83(14):7337-7348.

3. Burton DR, Poignard P, Stanfield RL, Wilson IA. Broadly neutralizing antibodies present new prospects to counter highly antigenically diverse viruses. Science. 2012;337(6091):183-186.

4. Kwong PD, Mascola JR. Human antibodies that neutralize HIV-1: identification, structures, and B cell ontogenies. Immunity. 2012;37(3):412-425.

5. Derking R, et al. Comprehensive antigenic map of a cleaved soluble HIV-1 envelope trimer. PLOS Pathog. 2015;11(3):e1004767.

6. Mascola JR, Haynes BF. HIV-1 neutralizing antibodies: understanding nature's pathways. Immunol Rev. 2013;254(1):225-244.

7. Derdeyn CA, Moore PL, Morris L. Development of broadly neutralizing antibodies from autologous neutralizing antibody responses in HIV infection. Curr Opin HIV AIDS. 2014;9(3):210-216

8. Willis JR, et al. Redesigned HIV antibodies exhibit enhanced neutralizing potency and breadth. JClin Invest. 2015;125(6):2523-2531.

9. Kong $\mathrm{R}$, et al. Improving neutralization potency and breadth by combining broadly reactive HIV-1 antibodies targeting major neutralization epitopes. J Virol. 2015;89(5):2659-2671.

10. Julien JP, et al. Crystal structure of a soluble cleaved HIV-1 envelope trimer. Science. 2013;342(6165):1477-1483.

11. Pancera M, et al. Structure and immune recognition of trimeric pre-fusion HIV-1 Env. Nature. 2014;514(7523):455-461.

12. Lyumkis D, et al. Cryo-EM structure of a fully glycosylated soluble cleaved HIV-1 envelope trimer. Science. 2013;342(6165):1484-1490.

13. Merk A, Subramaniam S. HIV-1 envelope glycoprotein structure. Curr Opin Struct Biol.
2013;23(2):268-276.

14. McLellan JS, et al. Structure of HIV-1 gp120 V1/ V2 domain with broadly neutralizing antibody PG9. Nature. 2011;480(7377):336-343.

15. Pancera M, et al. Crystal structure of PG16 and chimeric dissection with somatically related PG9: structure-function analysis of two quaternary-specific antibodies that effectively neutralize HIV-1. J Virol. 2010;84(16):8098-8110.

16. Walker LM, et al. Broad neutralization coverage of HIV by multiple highly potent antibodies. Nature. 2011;477(7365):466-470.

17. Trkola A, et al. Cross-clade neutralization of primary isolates of human immunodeficiency virus type 1 by human monoclonal antibodies and tetrameric CD4-IgG. J Virol. 1995;69(11):6609-6617.

18. Wu X, et al. Rational design of envelope identifies broadly neutralizing human monoclonal antibodies to HIV-1. Science. 2010;329(5993):856-861

19. Huang J, et al. Broad and potent neutralization of HIV-1 by a gp41-specific human antibody. Nature. 2012;491(7424):406-412.

20. Blattner C, et al. Structural delineation of a quaternary, cleavage-dependent epitope at the gp41-gp120 interface on intact HIV-1 Env trimers. Immunity. 2014;40(5):669-680.

21. Scharf L, et al. Antibody 8ANC195 reveals a site of broad vulnerability on the HIV-1 envelope spike. Cell Rep. 2014;7(3):785-795.

22. Huang J, et al. Broad and potent HIV-1 neutralization by a human antibody that binds the gp41gp120 interface. Nature. 2014;515(7525):138-142.

23. Walker LM, et al. Rapid development of glycanspecific, broad, and potent anti-HIV-1 gp120 neutralizing antibodies in an R5 SIV/HIV chimeric virus infected macaque. Proc Natl Acad Sci U S A. 2011;108(50):20125-20129.
24. Mikell I, Sather DN, Kalams SA, Altfeld M, Alter G, Stamatatos L. Characteristics of the earliest cross-neutralizing antibody response to HIV-1. PLoS Pathog. 2011;7(1):e1001251.

25. Klein F, et al. Somatic mutations of the immunoglobulin framework are generally required for broad and potent HIV-1 neutralization. Cell. 2013;153(1):126-138.

26. Pejchal R, et al. Structure and function of broadly reactive antibody PG16 reveal an $\mathrm{H} 3$ subdomain that mediates potent neutralization of HIV-1. Proc Natl Acad Sci U S A. 2010;107(25):11483-11488.

27. Briney BS, Willis JR, Crowe JE Jr. Human peripheral blood antibodies with long HCDR3s are established primarily at original recombination using a limited subset of germline genes. PLoS One. 2012;7(5):e36750.

28. Julien JP, et al. Asymmetric recognition of the HIV-1 trimer by broadly neutralizing antibody PG9. Proc Natl Acad Sci U S A. 2013;110(11):4351-4356.

29. Julien JP, et al. Broadly neutralizing antibody PGT121 allosterically modulates CD4 binding via recognition of the HIV-1 gp120 V3 base and multiple surrounding glycans. PLoS Pathog. 2013;9(5):e1003342.

30. Das R, Baker D. Macromolecular modeling with rosetta. Annu Rev Biochem. 2008;77:363-382.

31. Doria-Rose NA, et al. Developmental pathway for potent V1V2-directed HIV-neutralizing antibodies. Nature. 2014;509(7498):55-62

32. Kelsoe G, Verkoczy L, Haynes BF. Immune system regulation in the induction of broadly neutralizing HIV-1 antibodies. Vaccines (Basel). 2014;2(1):1-14

33. Liu M, et al. Polyreactivity and autoreactivity among HIV-1 antibodies. J Virol. 2015;89(1):784-798. 